\title{
High quality two-dimensional photonic crystal slab cavities
}

\author{
Tomoyuki Yoshie, ${ }^{a)}$ Jelena Vučković, and Axel Scherer \\ Department of Electrical Engineering, California Institute of Technology, Pasadena, California 91125 \\ Hao Chen and Dennis Deppe \\ Microelectronics Research Center, Department of Electrical and Computer Engineering, \\ The University of Texas at Austin, Austin, Texas 78712-1084
}

(Received 18 September 2001; accepted for publication 24 October 2001)

\begin{abstract}
We have fabricated and characterized donor-mode nanocavities formed by a single defect cavity defined within a two-dimensional photonic crystal slab. Quantum dots emitting in the 1.1-1.3 micron range were used as luminescence sources, and a design using fractional edge dislocations was used to demonstrate well-confined dipole modes with high quality factors. By applying the fractional dislocation geometry, the measured quality factor could be increased to values as high as 2800. This compares with typical quality factors of around 1500 measured from more conventional shallow donor mode cavities with larger mode volumes. (c) 2001 American Institute of Physics. [DOI: $10.1063 / 1.1427748]$
\end{abstract}

The combination of high quality factors $(Q)$ and small mode volumes are two important attributes for high performance nanocavities. High $Q$ microcavities will become very important for control of optical emission processes $^{1}$ for strong coupling experiments and many other industrial applications of novel light emitters. State of the art lithography and etching enables us to construct microcavities with many different kinds of geometries such as micropillars, ${ }^{2,3}$ microdisks, ${ }^{4} \mathrm{AlO}_{x}$-apertured vertical cavities,${ }^{5}$ and photonic band-gap cavities. ${ }^{6}$ Photonic crystal nanocavities ${ }^{7-9}$ have recently attracted much attention because such cavities are predicted to provide the desired combination of both small mode volume and high $Q$ values. Our photonic crystal cavities are constructed in thin slabs, which are perforated with triangular hole arrays and used to define photonic crystal mirrors. In the simplest defect cavity designs, regardless of whether we increase the number of defects forming the cavity, it has so far not been possible to demonstrate both small mode volume and high $Q$. The maximum $Q$ for donor cavities is around 1500 from extended modes in empty lattice photonic crystal cavities. ${ }^{10}$ Previous work to obtain higher $Q$ values has focused on thin slabs patterned within hexagonal hole patterns missing several holes within a photonic crystal lattice. However, those cavities are not suitable for many applications since they have relatively large mode volumes and many modes exist within the semiconductor emission range. Typical well-confined donor mode cavities have small mode volumes, but also suffer from relatively low $Q$ values, which are limited by vertical scattering losses. To address the problem, Vučković et al. ${ }^{11}$ recently predicted by 3D-FDTD (finite difference time domain) calculations that single defect cavities with fractional edge dislocations can have welllocalized modes with surprisingly high $Q$ values of as high as 30000 . In this letter, we report the experimental demonstration that by using fractional edge dislocations in photonic crystal cavities, it is possible to measure microcavities with high $Q$ 's as well as small mode volumes.

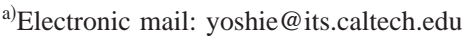

In Fig. 1, we describe the structure used in this work. The schematic of our single defect photonic crystal nanocavity is constructed within a thin slab of GaAs, which is perforated with a triangular lattice of holes. A single smaller hole replaces a larger hole within the center of this slab to define the optical cavity. This cavity supports doubly degenerate shallow donor states. In our design, we have modified this geometry in two important ways: (1) In the $x$ direction, in line with the smaller hole, ellipsoids rather than circles are fabricated at lattice spacing $(a)$ with an elongated major axis of $2 r+s=2 r\left(1+p / 2 a^{\prime}\right)$ where $r$ is a radius of original hole, $a^{\prime}=\sqrt{3} a / 2$ is an original distance of vicinal lattice array in $y$ direction, and $p / a$ is an elongation parameter. (2) Holes are moved away from the $x$ axis by $p / 2$ toward $+y$ or $-y$ direction. In $+y$ or $-y$ direction, the minor axis of ellipsoids is still $2 r$. These two operations correspond to adding a fractional edge dislocation in the $y$ direction. It also should be noted that Vučković et al. ${ }^{11}$ used the major axis of ellipsoids of $2 r+p$ which is bigger than the value $2 r\left(1+p / 2 a^{\prime}\right)$ used in this letter.

We used self-assembled grown quantum dots as the light-emitting material grown on (001) GaAs by molecular

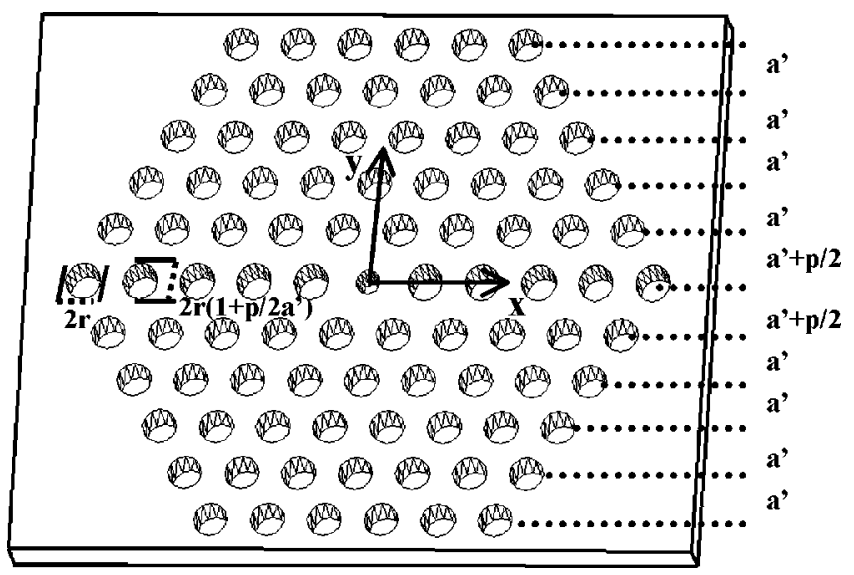

FIG. 1. Schematic of two-dimensional photonic crystal slab cavity with fractional edge dislocation. 

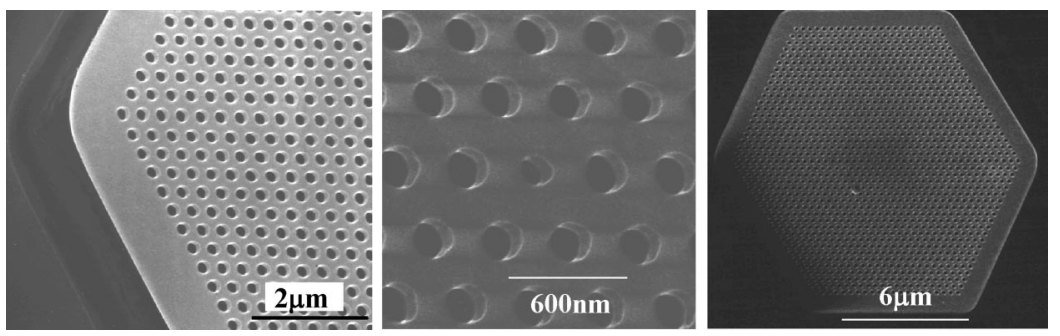

FIG. 2. Scanning electron microscopy image of photonic crystal slab with fractional edge dislocation.

beam epitaxy. Three stacked InAs quantum dot (QD) layers (QDs density: $3 \times 10^{10}$ dots $/ \mathrm{cm}^{2}$ ) were clad by $\mathrm{Al}_{0.16} \mathrm{Ga}_{0.84} \mathrm{As}$ layers on top of a $400 \mathrm{~nm} \mathrm{Al}_{0.94} \mathrm{Ga}_{0.06}$ As layer. A GaAs cap layer is then added to protect the top on the final layer. The cavity thickness $(d)$ is $240 \mathrm{~nm}$. The ground state emission of QDs used in this report showed linewidths as narrow as 40 $\mathrm{meV}$. The ground state emission at room temperature is peaked around $1240 \mathrm{~nm}$.

The patterns to form hexagonal-arrayed photonic crystal defect cavity ${ }^{12}$ were lithographically defined. Photonic crystal cavities are surrounded by twenty layers of photonic crystal for good optical confinement in plane. The lattice spacing (a) used in this work is lithographically controlled to 370 $\mathrm{nm}(d / a=0.65)$. After lithography, the beam-written patterns were transferred through the active membrane by using an $\mathrm{Ar}^{+}$ion beam assisted with a $\mathrm{Cl}_{2}$ jet, and the $\mathrm{Al}_{0.94} \mathrm{Ga}_{0.06} \mathrm{As}$ layer under cavities was subsequently oxidized in steam to define a perforated dielectric slab structure on top of an $\mathrm{AlO}_{x}$ cladding layer. Then, the $\mathrm{AlO}_{x}$ layer was completely dissolved in potassium hydroxide solution. Figure 2 shows images taken by scanning electron microscopy (SEM) for photonic crystal cavities containing a single donor defect with fractional edge dislocations. We fabricated samples with four different $p / a$ values $(p=0,0.10,0.15$, and $0.20 a$ ). The value of the hole radii defining the photonic crystals $r$ ranged from 0.28 to $0.29 a$, whereas the radius of the smaller hole defining the cavity ranged and from 0.20 to $0.23 a$. In the four samples, the geometry parameters have slight fluctuations, but the imperfections could be measured by careful SEM characterization and compared to model results.

Local microphotoluminescence pumping of the center of cavity, and measuring the luminescence normal to the sur-

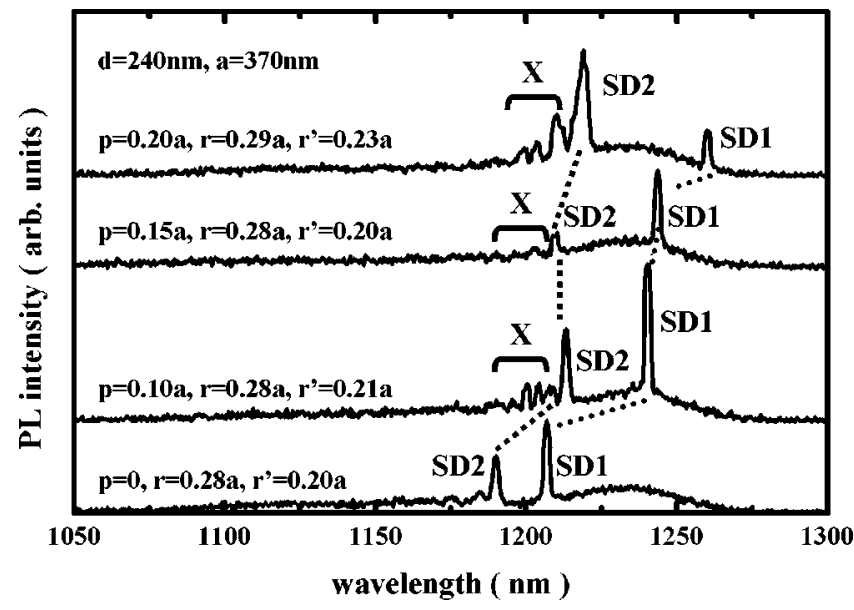

FIG. 3. PL spectrum of samples with different elongation parameter $(p / a)$ ranging from 0 to 0.20 .

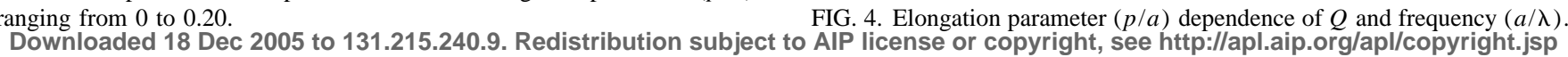

face, allows us to quickly compare the performance of different cavity designs. The $830 \mathrm{~nm}$ pump laser light was absorbed by the InAs quantum dots and the wetting layers, and the pump light could be focused onto the sample with a spot diameter of $2 \mu \mathrm{m}$. Light emission from the photonic crystal slab surface was then detected by an optical spectrum analyzer. The peak pumping power was $1.4 \mathrm{~mW}$, which corresponds to a power density of $45 \mathrm{~kW} / \mathrm{cm}^{2}$.

Figure 3 shows the $p / a$ dependence of photoluminescence spectrum measured from four cavities with almost the same $r / a$ and $r^{\prime} / a$. In this case, we selected a $2 \mathrm{~nm}$ bandwidth for the spectrum measurement to detect weak emission from the samples. The shallow donor (SD1 and SD2) peaks have distinct $y$ and $x$ polarizations, respectively. By considering polarization dependence and simulation results, SD1 and SD2 were found to match numerically predicted shallow donor modes. We believe that the degeneracy of shallow donor modes for $p / a=0$ are lifted because of fabrication fluctuations in our quantum dot nanocavities. In Fig. 3, it can be observed that, for $p / a=0$, only two donor modes are seen, whereas a group of peaks labeled as (X) emerge just above $\mathrm{SD} 2$ resonance in cavities with higher $p / a$ asymmetries. All the peaks shift toward longer wavelengths as $p / a$ increases. However, the shift of SD1 resonance seems to be more pronounced than that of SD2 resonance. This can be understood by assuming that the added edge dislocation overlaps better with the SD1 mode than with the SD2 mode. Figure 4 shows the influence of $p / a$ asymmetry on the $Q$ as well as the normalized resonance energy $(a / \lambda)$ taken from SD1 resonances. The SD1 resonance energy monotonically decreases almost linearly as $p / a$ increases for the same reason as described above. Though the shift is small, the SD2 frequency dependence also has the same trend. On the other hand, $Q$ continues to increase as $p / a$ increases for this plot. In our samples, a maximum $Q$ was obtained at $p / a=0.20$. Typical spectra taken from the nanocavities are shown in Fig. 5,

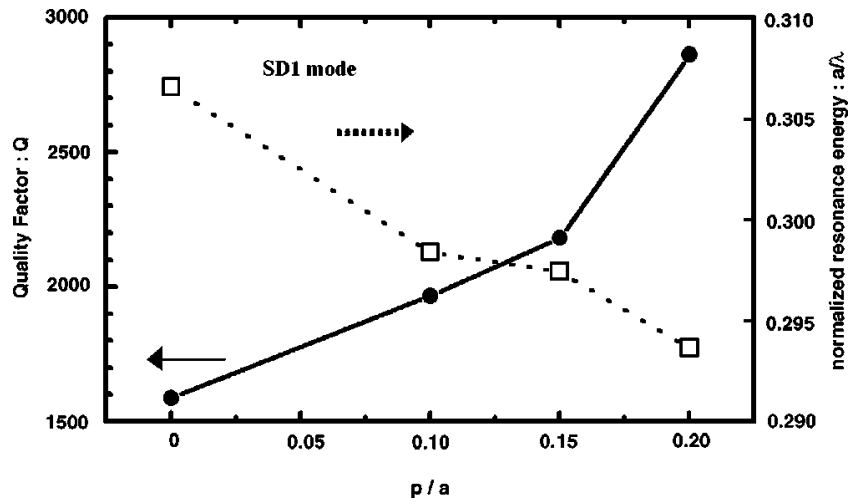




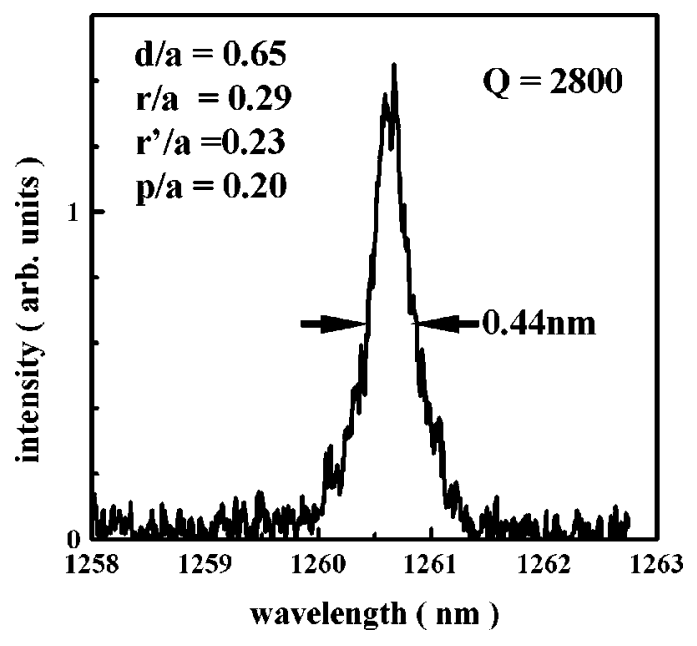

FIG. 5. PL spectra of the sample with $Q$ of 2800 .

where the resolution width of the spectrometer was set to 0.2 nm. The measured $Q$ was as high as 2800 . Therefore, by adding fractional edge dislocation, we could increase the measured $Q$ to twice the value measured from a symmetric cavity. To compare the measured $Q$ with our simulation results, we have carefully measured the geometries of our fabricated structures, and modeled these with our threedimensional FDTD code. Indeed, we find that the calculated $Q$ values for our experimentally realized geometries (of 4400) are much closer to the measured values, and that the simulated mode volume is $0.43(\lambda / n)^{3}$.

To confirm that the measured mode is localized to the defect region, we also changed the spatial location of pumping position to several points ten lattice layers away from the central hole. ${ }^{12}$ We could not see the SD1 resonance in such measurements, which leads us to believe that the shallow donor modes are well confined. This is a strong indicator that the measured cavity mode has both a high $Q$ as well as a small mode volume.

In order to verify whether the resonances appear in an appropriate range of emission, we have compared the observed spectra with spectra obtained by 3D-FDTD simulations, as shown in Fig. 6. For the simulation, spectral intensities in several points in the middle of the slab were added. Therefore, the spectra reflects on confined modes in the slab while the detected spectra reflects on leaky part of modes. That is why we also observe inhomogeneous emission from the QD layer in the spectrum. The SD1 and SD2 resonance frequencies are very similar to values predicted by the simulation. As the QD emission covers only the SD1, SD2, and air band region we did not see any prominent peaks around $a / \lambda=0.33$, which is shown in the simulated Fourier spectra. However, we believe those modes do not leak into air significantly since they are guided modes and the original intensity of the QD emission in this frequency range is too weak to be observed. Therefore, we conclude that the measured spectra showed a good agreement with the simulated one. Though our predictions indicate that $Q$ values of 30000 are possible in geometries similar with the ones presented here, our measurement results are enough to show the advantage of using the fractional edge dislocation over the conventional designs. We believe it will become feasible to obtain

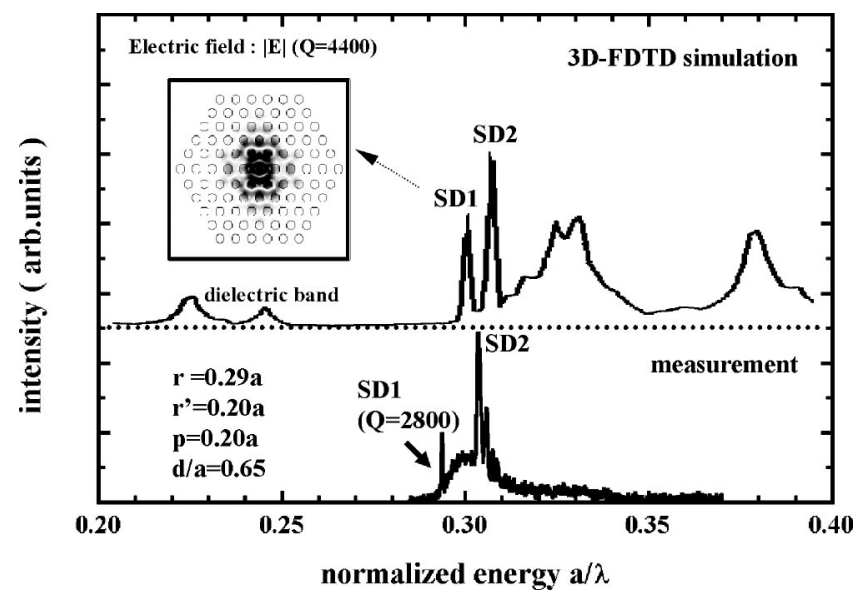

FIG. 6. Simulated Fourier spectrum of optical modes around a band gap.

the high $Q$ predicted once the optimum photonic crystal parameters are precisely fabricated, and we are presently working on more precise fabrication accuracy and similar designs to obtain even higher measured quality factors.

In summary, we have characterized donor modes supported in single defect nanocavities defined within, twodimensional photonic crystal slabs with fractional edge dislocations. The mode modulated by the dislocation was found to be well-confined around the single defect as predicted. High quality factors of up to 2800 were measured by luminescence from quantum dot emitters at $1.2 \mu \mathrm{m}$. These values were significantly higher than the values of 1500 measured for the simpler symmetric shallow donor mode cavities without the fractional edge dislocation. Our measured $Q$ values were limited by fabrication precision and fine design optimization, and these results indicate that further improvement of $Q$ is very likely in these kinds of geometries.

The authors gratefully appreciate many valuable suggestions from Marko Lončar. This work was supported by the NSF under Grant No. ECS-9912039, the DARPA under contract MDA972-00-1-0019, and the ARO under contract DAAD19-00-1-0374.

${ }^{1}$ E. M. Purcell, Phys. Rev. 69, 681 (1946).

${ }^{2}$ A. Scherer, J. L. Jewell, Y. H. Lee, J. P. Harbison, and L. T. Florez, Appl. Phys. Lett. 55, 2724 (1989).

${ }^{3}$ J. M. Gerard, D. Barrier, J. Y. Marzin, R. Kuszelewicz, L. Manin, E. Costard, V. Thierry-Mieg, and T. Rivera, Appl. Phys. Lett. 69, 449 (1996).

${ }^{4}$ A. F. J. Levi, R. E. Slusher, S. L. McCall, T. Tanbun-Ek, D. L. Coblentz, and S. J. Pearton, Electron. Lett. 28, 1010 (1992).

${ }^{5}$ D. G. Deppe, L. A. Graham, and D. L. Huffaker, IEEE J. Quantum Electron. 35, 1502 (1999).

${ }^{6}$ E. Yablonovitch, T. J. Gmitter, R. D. Meade, A. M. Rappe, K. D. Brommer, and J. D. Joannopoulos, Phys. Rev. Lett. 67, 3380 (1991).

${ }^{7}$ R. D. Meade, A. Devenyi, J. D. Joannopoulos, O. L. Alerhand, D. A. Smith, and K. Kash, J. Appl. Phys. 75, 4753 (1994).

${ }^{8}$ O. Painter, J. Vučković, and A. Scherer, J. Opt. Soc. Am. B 16, 275 (1999)

${ }^{9}$ O. J. Painter, R. K. Lee, A. Scherer, A. Yariv, J. D. O’Brien, P. D. Dapkus, and I. Kim, Science 284, 1819 (1999).

${ }^{10}$ J. Hwang, H. Ryu, D. Song, I. Han, H. Song, H. Park, Y. Lee, and D. Jang, Appl. Phys. Lett. 76, 2982 (2000).

${ }^{11}$ J. Vučković, M. Lončar, A. Scherer, and H. Mabuchi, Phys. Rev. E (in press).

${ }^{12}$ T. Yoshie, A. Scherer, H. Chen, D. Hufferker, and D. Deppe, Appl. Phys. Lett. 79, 114 (2001). 